This is the final peer-reviewed accepted manuscript of:

Marco Alvise Bragadin \& Kalle Kähkönen (2016) Schedule health assessment of construction projects, Construction Management and Economics, 34:12, 875-897.

The final published version is available online at: DOI: 10.1080/01446193.2016.1205751

Rights / License:

The terms and conditions for the reuse of this version of the manuscript are specified in the publishing policy. For all terms of use and more information see the publisher's website.

This item was downloaded from IRIS Università di Bologna (https://cris.unibo.it/)

When citing, please refer to the published version. 


\title{
Schedule Health Assessment of Construction Projects
}

\begin{abstract}
Several factors can contribute to the success of construction projects. A sound and good quality construction schedule is considered to be one of them. The quality of schedules has been a research topic only for a few efforts and amongst them construction oriented research is even more rare. Methodical grounds for assessing schedule quality has been studied via literature study for the development of appropriate solutions to assess the quality of construction schedules. These findings combined with the experiences from practical implementations has resulted in the definition of a metric to measure schedule quality for construction projects. It includes seventy five schedule requirements classified in five groups: general requirements, construction process, schedule mechanics, cost and resources and control process. This structure forms a core for the developed method to assess construction schedule quality termed as Schedule Health Assessment. The developed method has also the purpose of assisting project planners to produce and maintain good quality schedules starting from the project initiation until its completion, as via using the method to detect deficiencies of project schedules and other critical issues having importance with respect of schedule maintenance.
\end{abstract}

\section{Keywords:}

Construction management, scheduling, quality, project control, critical path method 


\section{Introduction}

Construction projects are generally complex endeavours, and project planning is an essential management function to guide construction project implementation, starting from the early design phase until project execution on site. Planning is a process of forecasting future events and outcomes that may be uncertain or even unknown. It means assessing the future and making provisions for it by gathering facts and opinions, in order to formulate an appropriate course of action (Uher, 2003). Project schedule is an important output of planning. Basically it explains the sequence of operations together with calendar dates and logic links between activities. Furthermore, a well-elaborated project schedule and model behind it can explain the dynamics of the project and can provide means for various analyses, project control and co-operation with project partners. Generally, the purpose of scheduling is to provide a guide that represents how and when the project will deliver the products defined in the project scope and by the project team (PMI, 2007).

Several factors can contribute to construction project success, and the schedule quality is considered to be one of them. A sound project schedule can be helpful in managing construction production with the purpose of improving productivity and quality through better planning and control. A good quality project schedule merge cost and technical data to support project management decision and actions, so project managers and stakeholders have to use project scheduling to understand project status and the probable development of future project activities. Therefore a good quality project schedule can be very important in the selection of an appropriate project organization form and of construction strategy (Russell, Tran, Staub-French 2014). Griffith (2005) and the guide of the United States Government Accountability Office 
(GAO) report that there is a significant relationship between good scheduling practices used early in the project life cycle and the ultimate success of the project (GAO, 2009). It looks obvious that the quality of the scheduling process and quality of the schedule itself can play an important role in the achievement of project success. Schedule quality survey can be thought as a key process of construction project management and an indicator of overall process quality (Zwikael and Globerson, 2004).

On the other hand the quality of construction schedules in particular has been researched in a rather limited manner. Previous research efforts that have addressed the content of schedule quality and its control with a direct approach, meaning with this an approach for a project control procedure, where the main attention is on the schedule audit with a contract management viewpoint, i.e. they have had a legal connotation and focus on contractual aspects, not on building site process (De La Garza, 1990, Moosavi and Moselhi, 2014). Obviously the quality of schedules, in general, has been always addressed in textbooks and scientific papers concerning construction project planning and scheduling methods and tools (Callahan et alii, 1992, Harris, 1978, O'Brian and Plotnick, 2006), and some industrial standards exists which cover procedures to achieve schedule quality, but most of those standards are outside construction context. Since construction projects can be considered as complex endeavours, and construction scheduling as process requiring then specific skills and competences, we are seeing this interplay as an object where a method for schedule quality assessment, or schedule quality evaluation can be beneficial for guiding the scheduling process. The leading idea is to create a pro-active method to develop and check the produced schedule. With this goal in mind a set of quality requirements applicable to construction schedules have been identified through pertinent literature and by exploring existing standards, and a metric to measure schedule quality is 
proposed for the purpose. The measurement system is based upon five KPIs, termed Schedule Health Indicators, derived from a categorization of the selected schedule requirements.

\section{Previous work}

Relevant information and data contained in a construction schedule require a proper reviewing process. O'Brian and Plotnick (2006) describe the reviewing process of a submitted CPM construction schedule with a legal accountability approach. In particular legal aspects are highlighted related to the consequences of the review process and to the rights of each part of the signed contract. After this, five major components of a good quality schedule are indicated: scheduling software; activity characteristics; network complexity; construction logic; dates and calendars. Each of these five major components focuses on specific check points or system requirements. Twenty four detailed check points are described. The aim of the review process is not to verify that the contractor can perform the contract work according to the plan of execution provided by the CPM, but that the project schedule is technically correct, and that logic and durations appear "reasonable".

Principles and characteristics of a quality-oriented scheduling process and of a quality schedule were also detected by De La Garza, East and Yau (1990) and De La Garza (1990). The study individuates three stages of the scheduling process which are needed for schedule analysis and validation:

- $\quad$ prior to the start of construction;

- during project execution; 
- at project completion.

In each of these stages a validation of the schedule is needed. The validation can be conducted by owners and contractors, and Project Managers and Schedulers need to check if the schedule meets the requested efficiency requirements. The validation process prior to the start of construction entails the check of the following schedule characteristics: contract requirements; participation of major subcontractors; inclusion of special construction oriented activities; critical path and overall degree of schedule criticality. The validation process during project execution entails: project control; schedule maintenance; detection of "in trouble" activities. The research does not address the third phase validation. An automated system called CRITEX, written for the U.S. Corps of Engineers has the purpose of "critiquing" construction schedules from four perspectives: general requirements, logic, cost and time of the project and of the activities. The system also encompasses 34 provisions for schedule quality assessment.

The contract management approach of O'Brian and Plotnick (2006), and of De La Garza et alii (1990) is believed to be a limit of the proposed schedule reviewing process.

Starting from these two main references, research based understanding of construction schedule quality has revealed three main research categories: characteristic of scheduling process, quality of scheduling process and quality of schedules.

Characteristic of Scheduling process is related to scheduling methods, rules and approach, focusing on the development of industrial standards about the scheduling function. A trustable scheduling process is about quality specifications of the production process for construction schedules, and schedule quality concerns the level of performance needed by a construction schedule (seen as a product). 
An industrial survey of CPM scheduling use in U.S. Construction Industry by Galloway (2005), revealed a remarkable need for standards in construction project scheduling.

Concerning industrial guidelines for project scheduling the first edition of the Project Management Institute "A Guide to the Project Management Body of Knowledge" (1996), defines the Knowledge Area of "Project Time Management" as a subset of Project Management that includes the processes required to ensure timely completion of the project. Project Schedule is simply defined as "the planned dates for performing activities and the planned dates for meeting milestones". Also the IPMA Competence Baseline (2006) includes in the Technical Competence elements the “Time \& Project phases", which entails a description of the possible process steps with a clear reference to the use of "Critical Path Diagrams". More recently, the APM Competence Framework of the Association for Project Management (2015) defines Schedule Management as "the process of developing and maintaining schedules for the work activities required to implement a change initiative",

The "Practice Standard for Scheduling" of the PMI (2007) confirms that a key to project success is to apply knowledge, experience and intuition to a project plan, and the attempt to execute according to the plan. Scheduling is one of the basic requirements of project management planning and strategic analysis, and has the purpose of providing a "roadmap" that represents how and when the project will deliver the products defined in the project scope. This can be achieved thorough a “Schedule Model" (PMI, 2007), a dynamic representation of the project's plan for 
executing the project's activities, developed by the project team's applying the scheduling method to a scheduling tool using project specific data such as activity lists and activity attributes. A scheduling method is a system of practices, techniques, procedures and rules used by project schedulers and performed either manually o with a project management software, i.e. a scheduling tool which provides schedule components supporting the application of a scheduling method (PMI, 2007). The Schedule development process includes selecting a scheduling method and tool, incorporating project specific data within that scheduling tool to develop a project specific schedule model and generating project schedule. This process has the aim of producing a schedule model of project execution, which has to be regularly updated to reflect progress and changes. Scheduling process includes activity definition, activity sequencing, activity resource estimating, activity duration estimating, schedule development, schedule control (PMI, 2007).

The AACE International (AACE) Recommended Practice No. 14R-90 (Douglas, 2006) describes the roles and responsibilities of a Planning and Scheduling Professional during the various phases of project planning and schedule development, management and control, also establishing a planning and scheduling guidelines for training and professional development. Scheduling is defined as the "process of converting a general outline plan for a project into a time-based schedule based on available resources and time constraints".

The GAO "Cost Estimating and Assessment Guide" (2009), describes recommended best practices for developing and managing capital program costs of projects using public funds. The guide focuses on project cost estimating, planning and managing. The GAO guide includes between major reasons of project success the quality of its schedule. Schedule provides a time sequence for the duration of project 
activities, and should integrate the logical relationship between activities, activity resources requirements and durations, and any constraint that affect their start and completion. The GAO guide indicates nine requirements useful to develop and maintain and integrated network schedule.

The CEN Workshop Agreement CWA 16022 (2009) "Project Schedule and Cost Performance Management (PSCPM) gives specific indications on the informative system entailed in project scheduling function.

\section{Quality of Scheduling Process}

Quality identification of scheduling process aims at developing the schedule production process in a way that the final product (the schedule) will have a set of inherent characteristics that will fulfil project requirements. The intended functionality of the schedule should be validated during the course of the project (De La Garza, 1990; De La Garza, East and Yau, 1990).

Zwikael and Globerson (2004) introduced a model for evaluating the quality of project planning called "Project Management Planning Quality (PMPQ)". The model consists of the two following components:

- project manager's know - how, including processes for which a project manager is responsible, derived from the PMBOK;

- organizational support offered by the performing organization.

The PMPQ model identify 16 know-how processes and output and 17 organizational support processes and products. The processes were grouped into knowledge areas, based on the PMI classification and on existing Project Management maturity model, 
9 Knowledge areas (from PMBOK) for Project Know-How and 4 Knowledge areas for Organizational support.

The model is structured to convert all these variables into an overall quality indicator. To achieve this purpose a relative importance, or weight, has been assigned to each process. Equal weight was assumed for the two groups, Know-How and Organizational, and also equal weight was assumed for each Knowledge Area. The weight of a specific process within a certain area depends on the number of processes in that area. The PMPQ index, that evaluates the quality of project planning process in the organization, is calculated as a weighted average of the 33 processes evaluation. But, indeed, the focus of the work is not on scheduling process and it is not construction oriented.

The Practice Standard for Scheduling of the Project Management Institute (2007) describes the schedule development process good practices and components. Key points in the schedule development process are needed for scheduling quality: schedule components and data; schedule development process activity definition; schedule model characteristics; project control features. The Scheduling Maturity Model (APM, 2012) has the aim of measuring an organisation's ability in implementing and applying a scheduling process, with the aim of producing a good quality and robust schedule. The Schedule Maturity Model can be used for the assessment of a single project schedule or to benchmark the quality of the scheduling process through the organization, and it is based upon the definition of 28 attributes, classified into 7 requirements: process and toolset; structure and hierarchy; integration; resource / cost integration; risk; update and maintenance, environment. 


\section{Quality of Schedules}

Quality of schedules entails requirements specification and performance metrics to define a quality schedule. Schedule quality assessment was the aim of the study of Russell and Udaipurwala (2000). Here the perspective on schedule quality assessment is related to construction strategy, "the plan of attack", plus the timing of activities. Russell identifies various indicators of schedule quality, grouped under several headings: accuracy and completeness, consistency with other planning documents, good practice/workability and benchmarks for control. Abstraction and compliance with contract documents are quoted but not examined in the cited literature.

AACE International (AACE) Recommended Practices (Douglas, 2006; Douglas and Gransberg, 2009) include the "Schedule Quality Analysis" and a guideline for schedule constructability. Schedule Quality Analysis means the checking of the schedule specification compliance, the verification of the schedule integrity (i.e. schedule mechanics and constructability) and the schedule validation. The guideline for schedule constructability, instead, entails a review process of a construction schedule, termed Schedule Constructability Review (SCR). The goal of SCR process is to assess whether the schedule is comprehensive and complete. Constructability can be defined as the use of construction knowledge and experience in planning, design, procurement and field operation to achieve overall project objectives (Douglas, 2006; Douglas and Gransberg, 2009).

The Practice Standard for Scheduling of the Project Management Institute (2007) defines a Conformance Index and a Conformance Index Assessment process to evaluate schedule quality. The GAO "Schedule Assessment Guide" (2012), describes recommended best practices for project schedules. The guide focuses on 
project schedule quality to help managers and auditors ensure that the project schedule is reliable. The GAO guide provides ten best practices associated with a high quality and reliable schedule.

The US Defense Contract Management Agency (DCMA) has defined a wellknown 14 points metrics aimed at identifying potential problem areas with a contractor's Integrated Master Schedule (DCMA, 2012). The DCMA 14 point schedule metrics is a tool that supports the schedule analysis to determine whether it is a realistic schedule or not, i.e. gives a metrics for assessing schedule quality. The schedule quality assessment can also be performed by an automated MS Project Macro developed by the agency.

The National Defense Industrial Association (NDIA), working group of Industrial Committee for Program Management, published the "Planning and Scheduling Excellence Guide (PASEG)" to provide the project management team, including new and experienced master planners/schedulers, with practical approaches for building, using and maintaining the project master schedules (2012). The guide encompasses "Generally Accepted Scheduling Principles (GASP), 10 quality control steps to validate the Integrated Master Schedule and a list of metrics that can be used to assess schedule health. The PASEG guide first introduces the term "Schedule Health Assessment" as a quality control of project schedule and suggests the implementation of an automated schedule health assessment tool. The PASEG approach of Schedule Health Assessment is different from the procedure proposed in the following paragraphs, as it focuses only on the "mechanics" of the schedule, and it is not construction oriented. The same observation can apply also to the previously cited guides and metrics. 
Moosavi (2012) and Moosavi and Moselhi (2012, 2014) defined a structured methodology to assist owners in the evaluation and approval of detailed schedule of contractors. In essence it is a check list that covers a set of overall requirements for good schedules, concerning both development process and final schedule. The methodology has been implemented in an automated computer application called "Schedule Assessment and Evaluation - SAE" developed to assist owners in the review of project schedules. The SAE performs schedule evaluation in three tiers:

1. Assessment of the schedule against industry recommended practices;

2. Job logic assessment of construction trades;

3. Assessment of construction productivity and of crew size considered for a number of commonly used trades in building construction.

The method is based on the evaluation of forty eight criteria for schedule health assessment including conceptual provision as well as quantitative requirements. The criteria are divided into three major categories: contractual compliance, schedule development, and schedule components. In the first step of the research (Moosavi 2012) the criteria were classified in conceptual provisions and quantitative provisions, actually they are classified in eight obligatory criteria and forty complementary criteria (Moosavi and Moselhi, 2014). The contractual management approach is believed to be a limit of the SAE method.

\section{Research methods}

The definition of the characteristics of a good quality schedule can be a challenging task. However some experts from academia and professional institutions have 
proposed planning standards and schedule quality assessment methods. The seminal work of De La Garza (1990) and the consequent research and standardization efforts, indicated the way forward for a construction schedule quality assessment. From this starting point, and passing through the definition of Project Management and Planning Maturity Model (Zwikael and Globerson, 2004), a research line has been developing from various and different roots, citing before all the works of Birrel (1980) and Laufer and Tucker (1987), which aim both at creating the conditions of producing a good construction schedule, thought as a symbolic tool of the planning effort of the project management team. The Schedule Management Maturity Model of APM (2012) is a step forward in this direction. The main idea is not only to measure schedule adequacy, but also to indicate the processes, the phases and the working environment needed to create a robust schedule.

With this perspective, a method for construction quality assessment has been developed, with the aim of being also a guide for project schedulers in the scheduling process. Actually, almost all existing guidelines and standards about schedule quality are control oriented, and do not sufficiently highlight the schedule development phase. Moreover few considers the building and construction dimension of the production process and most of them have a strong legal accountability connotation.

First, it is thought that construction process and technology knowledge is of paramount importance in schedule management and development, and the proposed method highlights construction process requirements of project scheduling. This is seen to be a relevant contribution of the proposed Schedule Health Assessment approach. Then, another contribution is the aim at creating a guide for the project schedulers to be used in the design phase and for updating during project work execution. 
The research methods followed are the constructive research approach (Lukka, 2000; Lukka and Kasanen, 1995) and this producing a proof of concept experiment for the feed-back phase. Firstly the relevant requirements needed for the development of an adequate construction schedule have been detected from the pertinent literature. Secondly a thorough understanding of the applicable requirements was obtained by sample applications on project schedules. The solution idea proposed was to create a schedule management approach to qualify, guide and control the scheduling process. The approach can be used in both cases, the one of owner's consultant or the one of a project scheduler of a Construction company. As small companies are expected to have more resource problems (Hussain and Werne, 2005), the proposed method can be used as a facilitator of the scheduling process in the very common case of small - medium construction company projects. The constructed procedure was termed Schedule Health Assessment and it was developed with a simple set of score sheets to record the quality analysis performed. The evaluation of the quality level achieved by the construction schedule is performed through five Key Performance Indicators (KPIs), termed Schedule Health Indicators. Thus the research is believed to produce an innovative "construction" meant to solve the initial real-world problem of low quality level of construction schedules (Lukka, 2015). The developed method can also offer a conceptual framework of the scheduling problem for construction in general.

The research work develops in three steps (figure 1). The first step is inductive and addresses the analysis of all the schedule quality requirements defined by researchers and by international standards or recommended practices. One hundred and fifty-six specific schedule quality requirements have been evaluated and classified from pertinent literature. 
The second step involves the selection of a group of seventy five requirements divided into in five classes of requirements. The selection process was performed identifying all pertinent requirements found in literature, and grouping them into classes of requirements. After this the developed method performs the evaluation of the construction schedule quality thorough detailed requirements' check lists. A weighted approach allows to assess the global "health" of the evaluated schedule.

In the third step the Schedule Health Assessment proposed procedure is tested on a sample case study, concerning both the planning and the controlling phase. The case study offers the possibility of proof of concept and generalized conclusions (Lukka, Kasanen, 1995), because of the possibility to discuss specific results and the subject area of construction scheduling in general.

$<$ Please insert figure 1 here $>$

\section{Schedule Health Assessment: Quality Indicators}

\section{Main components of construction schedule quality}

Many components are required in the development of a good quality project schedule, but two of them are believed to be more relevant to create a construction oriented scheduling model, as described by literature. Construction schedule quality is, indeed, the result of the interaction between two main components, construction knowledge transferred into project schedule and schedule mechanics, the latter meaning the mathematical-related part of the schedule, mainly inherent to network logic and time computation. The assumption is that a standard construction schedule 
is basically prepared applying well-known networking methods like Precedence Diagramming Method, and using a software application such as MS Project ${ }^{\circledR}$ or Primavera P6®. By construction knowledge it is meant the set of information related to construction technology implementation in the building construction process, while by schedule mechanics knowledge it is meant the set of information related to scheduling technology, ie scheduling and activity network rules. Actually construction knowledge implementation refers to the constructability concept, as previously addressed, i.e. a system for achieving optimum integration of construction knowledge and experience in planning, engineering, procurement and field operations in the building process, and balancing the various project and environmental constraints to achieve overall objectives (IPENZ, 2008). Actually constructability concept can be useful for quality assessment to review construction processes from start to finish during pre-construction phase. The aim of constructability check is to identify obstacles before the project is actually built to reduce or prevent errors, delays, and cost overruns.

Instead schedule mechanics knowledge refers to the project management methods and techniques to plan and schedule a project. As most of construction projects are scheduled with a networking technique, e.g. Precedence Diagramming Method, implemented with a computer software, schedule mechanics is the set of rules that allows the performance of the scheduling process with a critical path method on a computerized application. While in most standards, recommended practices and pertinent literature construction knowledge and schedule mechanics knowledge are addressed with a separate approaches, it is believed that an integrated approach could be more effective for a construction schedule. Two specific quality indicators of the proposed method are dedicated to the assessment of these two areas of knowledge. 
Construction knowledge assessment mostly depends on activity definition and on structure of network logic. These and other important requirements can be found in the Indicator no. 2 (table A2). Schedule mechanics knowledge mostly depends on network logic structure and critical path time analysis, as described by the indicator no. 3 (table A3). The proposed schedule quality assessment procedure has been termed as Schedule Health Assessment, as suggested by the PASEG guide in 2012 (PMSC, 2012), because it is felt that this denomination quickly brings to the reader the concept of good quality, good health indeed, of the schedule as a part of the project management process. But it is also felt that the PASEG guide is too much a generic guide as a starting point for construction schedule evaluation process, because it is not construction oriented and focuses mostly on schedule mechanics (and activity definition and network structure). The most important quality indicators, or health indicators, should be related to construction knowledge and schedule mechanics knowledge, but this is not enough. Quality indicators should also describe all the components of schedule quality, and must concern with scheduling process itself, with cost and resource data, and with the control process performance. So the developed quality assessment procedure is based on the identification of five quality indicators or KPIs, and on the measure of their values. Each schedule indicator aims at defining a quality level of schedule performance concerning a specific component thus contributing to assess overall schedule health. 
The structure of the proposed method is based upon five Health Assessment Indicators of schedule quality. These indicators have the task of measuring the performance of the scheduling process and of the produced schedules. Each indicator is composed by different classes of requirements, simply termed requirements which in turn are made up of detailed requirements, being the "measurement items" of the method. The method originates from a literature analysis in which 156 different detailed quality requirements for scheduling have been detected. These detailed requirements have been used as background data, they have been classified, analysed and grouped depending on their specific subject, content and purpose. The classification of these requirements was performed in three steps. In the first step all the found literature requirements have been listed and understood. In the second step the literature requirements have been classified using candidate categories for the targeted schedule quality assessment system. This resulted in the five Schedule Health Indicators, which were further divided into classes of requirements. In the third step the selection process has been finalised with the following criteria: unicity of the specification (i.e. avoiding repetitions); level of importance related to the aim of the research; usefulness in the construction sector. The requirement list has been improved by adding a construction safety detailed requirement. This process produced seventy-five detailed requirements, which are classified in five groups to constitute the five different Schedule Health Indicators.

The identified five Schedule Health Indicators are the following (fig. 2):

1. General requirements;

2. Construction process requirements; 
3. Schedule mechanics requirements;

4. Cost and resources requirements;

5. Control process requirements.

The objectives and aims of each Schedule Health Indicator are the following.

- Indicator no.1 "General requirements" consists of a set of provisions that are aimed at conforming the schedule production process to quality standards related to the developing phase, to the schedule as a product, and to the contract provisions for the construction project (appendix: table A1).

- Indicator no. 2 "Construction process requirements" consists of a set of provisions that are aimed at conforming the schedule to quality standards related to the execution phase of the construction project. The objective is the construction process safety and optimization (appendix: table A2).

- Indicator no. 3 "Schedule mechanics requirements" consists of a set of provisions that are aimed at conforming the schedule to quality standards related to the planning/monitoring phase of the construction project with the critical path method (appendix: table A3).

- Indicator no. 4 "Cost and resources requirements" consists of a set of provisions that are aimed at verifying that the activities of the project, and the project itself, can be executed within the calculated time and budget, due to resource and cost loading to activities (appendix: table A4).

- Indicator no. 5 "Control process requirements" consists of a set of provisions that are aimed at allowing an efficient project control process through schedule updating and re-planning processes (appendix: table A5). 
$<$ Please insert figure 2 here $>$

Each indicator is, in turn, composed by a number of requirements aimed at the quality assessment of the produced schedule, and at developing a construction project schedule conforming to the suggested method.

The information content of each indicator is first expressed by "Requirements", than each of them is composed of more specific "Detailed Requirements", which, in turn (and if needed) are composed by "Requirement Specifications". The method classify twenty-one requirements, seventy-five detailed requirements and fifty-four requirement specifications (Bragadin, Kahkonen, 2014).

The schedule quality measurement process is organized through a Breakdown Structure (fig. 2). First detailed requirements with optional requirement specifications are checked. The satisfaction of detailed requirements gives a measure of each Schedule Health Indicator satisfaction. Then each indicator is weighted and a measure of total schedule quality can be found. A brief description of each Schedule Quality Indicator and its component requirements is following. The complete list of detailed requirements is included in the appendix to the text (tables A1, A2, A3, A4, A5).

\section{The General Requirements Indicator}

The General Requirements Indicator (figure 2) is composed by three requirements: Schedule Process Procedure; Schedule Definition; and Activity definition. The Schedule Process Procedure requirement aims at conforming the schedule 
development process to four related quality standards, namely detailed requirements: definition of an activity coding structure; identification of project calendars; involvement of main subcontractors and following of a standardized scheduling procedure. The Schedule Definition requirement entails five more detailed requirements about schedule structure and contract compliance. In particular it concerns schedule logic vertical and horizontal integration, meaning that detailed tasks must flow-up to summary tasks and there must be logical relationships and timephasing between tasks. The Activity Definition requirement encompasses detailed requirements mainly about the total number of activities, activity name and definition, WBS, and responsibility assignment (appendix: table A1).

The Construction Process Requirements Indicator

Four are the requirements composing the Construction Process Requirements indicator: Activity Sequencing \& Structure adequacy; Activity Duration, Activity Timing and Construction Process Safety and Productivity (figure 2). They are mainly concerned with schedule constructability, meaning the proper sequence of construction phases and the optimisation and safety of the construction process modelled by schedule. Activity Sequencing aims at defining a construction-oriented network logic, while Activity Duration is related to the duration estimate and the continuity of production during activity execution. Activity Timing detailed requirements are mainly related to proper scheduling of weather sensitive activities. Construction Process Safety and Productivity is related to work efficiency of the 
construction site, and entails detailed requirements about work continuity, work flow of resources, work space and safe work areas (appendix: table A2).

\section{The Schedule Mechanics Requirements Indicator}

Schedule Mechanics Requirements Indicator aims at conforming the network schedule structure to critical path method related detailed requirements (figure 2). So the indicator is composed by detailed requirements related to network and logic, critical path and critical activities, float dimensions and computation, soft and hard constraints, buffers, leads and lags, activity mis-assignments (appendix: table A3). Schedule mechanics requirements are believed to have an high impact on schedule quality. Network and logic requirement aims at conforming the PDM network structure to requested features in terms of absence of open-ended activities, proper use of summary task logic, no activity with missing predecessor or successor, a correct number of relationship between activities, frequent use of finish-to-start relationship types, and few activities with an high duration. Critical path detailed requirements are bound to properly define critical path logic and critical activities of the network, and also entail few quantitative indicators as the Critical Path Length Index (CPLI), Schedule Criticality Rate and Near Criticality Rate, as proposed by the DICMA 14 points standard. Float requirements are related to float computation and dimension. Excessive total float and negative total float are discouraged. Soft and Hard Constraints and Buffer detailed requirement entail ASAP and ALAP computation, the type and number of constraints and the presence of buffers. As defined by Activity Mis-assignment and Empty Event detailed requirements, no one of such activity or event must be found in 
the network. Lead and Lags provide a small amount of relationships with lead times, and no relationships with negative lead, termed lag.

\section{The Cost and Resources Requirements Indicator}

Cost and Resources requirements indicate the feasibility of the construction schedule as they implicate the effective amount of economic and working resources (figure 2). The indicator is composed by four requirements: Monetary Value / Cost of activities, Project Cost Ratio, Resource Loaded Activities, Project Total Level of Effort (appendix: tab. A4). The Monetary Value / Cost requirement entails the monetary load of activities in terms of cost or prices of all the activities, the total monetary value of the project and the optimized distribution of progress payments, including the Project Cost Ratio Index. The Resource Loaded Activities requirement detect the resource loading of activities, the planned productivity which has contributed to the duration computation, and the possibility of resources conflicts. Project Total Level of Effort and Project Effort Ratio can be important markers of the schedule feasibility.

\section{The Control Process Requirements Indicator}

Control Process requirements have the task of facilitating Project Control activities. The four requirements defined are the following: Activity Progress Evaluation, Schedule Review and Baseline, Schedule Projections and Invalid Dates and Missed Tasks (figure 2 and appendix: tab. A5). Activity Progress Evaluation is composed by 
two detailed requirements: the percentage complete of activities and schedule slippage. The Schedule Review and Baseline requirement has the aim of evaluating the schedule maintenance process, and entails actual progress definition, the report of variances and the Baseline Execution Index (BEI), the latter being of major importance for the evaluation of the actual progress of work related to schedule forecast (DCMA, 2012). Schedule Projections requirement entails two detailed requirements: schedule projections and corrective actions. These two items aim at revealing the implementation of schedule updating and control actions. Control process requirements list ends with the invalid dates, missed tasks and out of sequence detailed requirement, which all are well known indicators of the accuracy of schedule updating.

\section{Schedule Health Assessment}

\section{Schedule Health Assessment proposed method}

Schedule Health Assessment is a method to perform the quality assessment of a construction schedule. The aim is to ensure the quality of the construction schedule starting from its design and development phase, through the maintenance phase during project work execution until project completion. Beyond the intended use for quality control purposes, the developed Schedule Health Assessment procedure also aims to assist project team and project schedulers to prepare a good quality schedule which fits the needs of project owner, contractors and stakeholders. It is, indeed, a procedure that checks the quality of the construction schedule which can be used by the contractor 
in the development and maintenance of the project schedule or by the owner in the evaluation and review of the schedule submitted by the contractor.

The Schedule Health Assessment proposed method also produces a report containing a set of data or statistics reviewed for compliance up to a set of seventyfive requirements belonging to five schedule KPIs termed Schedule Health Indicators. Schedule Health Metrics are different from schedule execution metrics, as they focus on schedule adequacy, while schedule execution metrics focus on the performance of project work processes (NDIA, 2012). For instance, in the Schedule Health Assessment procedure it is evaluated if a Baseline Execution Index has been computed, and not if it has a favourable or unfavourable value or, generally speaking, if the project execution is running in line with forecasted performance or not.

The construction schedule quality model

The Schedule Health Assessment proposed method uses seventy-five detailed requirements to constitute a metrics to measure schedule compliance to a quality model of the process/product "construction schedule". The quality model was built through study and analysis of pertinent literature and standards, and from suggestions from practical experiences. Each detailed requirement constitutes an item of construction schedule quality that can be a quality-related sub-process, i.e. "Main sub-contractors participation", or a quality-related component, a schedule feature or an item, i.e. "Critical path \& critical activities", "Relationship ratio" or "Monetary Value of Activities". 
Thus it is quite simple to evaluate if the quality-related sub-process has been performed by project team or if the quality-related component was included in the schedule. The evaluation is done by a checklist scoresheet where one point is earned if the detailed requirement check is positive (pass) or no points are assigned if the check is negative (fail). A similar evaluation process is proposed by the PMI for the Schedule Conformance Index (PMI, 2007). Concerning the evaluation of each single detailed requirement, if needed more specific "requirement specification" have been used to facilitate the evaluation process. "Requirement specification" list has not been included for brevity sake. Detailed requirements are grouped into five Schedule Health Indicators, and a Requirement Breakdown Structure (RBS) has been encoded to guide and facilitate the assessment process. An excerpt of the scoresheet used for the evaluation of quality of the sample case study schedule can be found in figure 3 .

$<$ Please insert figure 3 here $>$

Thus a quality assessment of the construction schedule can be performed with a rather simple and quick method. But for evaluation purposes the procedure needs to take into account the relative importance of each set of requirements to the global quality of the schedule. Indeed, the five Schedule Health Indicators do not have the same importance in planning and scheduling process. While some of the studies and recommended practices focus on the requirements related to constructability (De la Garza, 1990; Dzeng, Lee 1999; Douglas and Gransberg, 2009), which mainly correspond to the Schedule Health indicator no. 2 (Construction process requirements), most of guidelines and standards, (PMI, 2007; U.S. DICMA, 2012; 
PMSC - NDIA, 2012) highlight the importance of the scheduling process and product quality only addressing schedule development and mechanics, which correspond to Schedule Health indicators no. 1 and no. 3, (General requirements and Schedule mechanics requirements). Also Moosavi and Mosehli (2012) who performed a survey based on feedback from professionals of the construction industry, indicate as top schedule assessment criteria the ones related to the scheduling process and to schedule mechanics. Although the cost and resource loading requirements are believed to be fundamental components of the planning, scheduling and controlling processes, they seem to have less value for schedule quality assessment as described in pertinent literature. The same can be said for the project control requirements. Assuming that all the relevant requirements for quality evaluation of a construction schedule have been identified, there is a need to assign them a relative importance, a weight, to structure a quality assessment method (Paquin, Couillard and Ferrand, 2000).

Since there is no prior information concerning the relative importance of each detailed requirement belonging to each Schedule Health Assessment Indicator, they are assumed to have the same importance in their own group. But the number of detailed requirements of each Indicator seems to be in direct relationship to the level of relative importance of the Indicator related to the others, so the developed method weights each Schedule Health Assessment Indicator in function of the number of the composing detailed requirements. A similar logic was implemented to structure the weights of the model of Zwikael and Globerson (2004).

$<$ Please insert figure 4 here $>$ 


\section{Health Assessment Procedure}

The method identifies a number of measurable detailed requirements for each Schedule Health Indicator of the construction project schedule. Then quality evaluation of the schedule can be performed with a check list (figure 3 ) of detailed requirements satisfaction. With this information the quality level indicated by each Indicator can be assessed and a comprehensive quality level, the Schedule Health Assessment, can be evaluated through a weighting process. The procedure and results of the proposed method are shown in figure 4 , where a schedule performance chart is presented to show the results of the proposed procedure.

The overall Schedule Health $(\mathrm{SH})$ can be quantified with a percentage grade. For each Schedule Health Indicator (Si) the weight (Wgi) indicates the relative importance of each indicator to the others being used to measure the overall performance of the schedule of the construction project.

$$
S H=\sum_{i}^{5} S i \times W g i
$$

where $\mathrm{SH}=$ overall Schedule Health assessment of the construction project; $\mathrm{Si}=$ Schedule Health Assessment Indicator (i); Wgi = weight of Schedule Health Indicator (i), compared to other indicators of the schedule. The weight can vary related to the number of Indicators used for the evaluation. To highlight this process a specifying character can be added: $\mathrm{Wgmi}=$ relative weight for master schedule evaluation; Wgdi= relative weight for detailed schedule evaluation; Wgci $=$ relative weight for maintenance schedule evaluation (fig. 5). 
The model of equation (1) allows to measure and quantify the overall health of the construction project schedule.

The Schedule Health Assessment Indicator (Si) for the requirement group (i) of the scheduling process is evaluated with the percentage of adherence to detailed requirements and specifications for schedule quality. The indicator is calculated with the following equation (2):

$$
S i=\frac{\sum_{j}^{S} D R i j}{\sum_{j}^{S} R i j}
$$

Where $\mathrm{Si}=$ Schedule Health Assessment Indicator (i); DRij = estimated detailed requirements met by schedule (j) for the schedule health assessment indicator (i); Rij $=$ detailed requirements $(\mathrm{j})$ for the Schedule Health Assessment Indicator (i); s = total number of detailed requirement of the Indicator. Note that for each detailed requirement satisfaction the DRij value is 1 , if the schedule does not meet the requirement the DRij value is zero.

A pro-active method for construction schedule development

The Schedule Health Assessment proposed method has also the goal of supporting project planners in the development of a high quality project schedule. With this goal in mind Schedule Health Indicators have been put in a sequence thinking at their progressive implementation during the planning, scheduling and controlling process. The construction schedule development process can be implemented with three steps: 
master schedule, detailed schedule, schedule maintenance. The first and the second step form the planning phase, while the third step is the control phase.

a) Master Schedule. In the first step project schedulers can start schedule design and development following the general requirements. Then Master Schedule can be created following construction process requirements and schedule mechanics requirements. Once project schedule is developed, checklist scoresheets can be used to assess schedule health level through Indicators number one, two and three. This control step provides a feedback that can be used to improve schedule quality.

b) Detailed Schedule. In the second step cost and resources can be added to the construction schedule following cost and resources requirements. This process generally involves a more detailed definition of project activities. Then a control feedback can be performed mainly through quality evaluation of Indicator number four, but also indicators number one, two and three are considered again.

c) Schedule Maintenance. When project execution phase commences, the control process of project execution can start and the updating and maintenance processes of schedule can follow the control process requirements' track. Mainly the quality evaluation is performed with the checklist scoresheet of Schedule Health Indicator number five. This can give a useful control feedback to project managers and schedulers, highlighting schedule updating items to improve. In the schedule maintenance phase, indeed, re-planning and rescheduling are performed and all of the five indicators are needed. 
The proposed three steps for construction schedule development and quality checking allow project scheduler to perform the proposed Schedule Health Assessment method related to the needed level of schedule application. Weights of indicators vary depending on the level of implementation. In fact for real life construction projects a partial schedule development is very common, i.e. only phase one, or one and three can be fully implemented (figure 5).

$<$ Please insert figure 5 here $>$

The proposed procedure can be easily performed by the project scheduler during each scheduling phase analysing schedule features, reviewing the file(s) produced by the scheduling software and completing the proposed checklists. It is possible to modify weights from project to project based on project priority. As the proposed method basically consists of five checklists (one for each indicator), it is believed that completing the checklist is an easy task for the project scheduler. The procedure was tested on the sample project schedule following, but has been already tested on another schedule in a previous paper (Bragadin, Kahkonen, 2014).

\section{Schedule Health Assessment implementation and sample testing}

The developed Schedule Health Assessment method has the goal of being simple and easy to be applied by practitioners. So the evaluation process can be performed easily by the project scheduler assessing schedule performance as measured by the requirements specified in the checklists. Note that the requirement lists can be 
emended to fit to specific project needs. The Schedule Health Assessment procedure can be accomplished in a straight forward manner. First the detailed requirement check list is evaluated. Each Schedule Health Indicator (e.g. General requirement) is composed by requirements (e.g. Schedule Process Procedure, Schedule Definition, Activity Definition). Each requirement is made up of various detailed requirements, as previously defined. The scheduler checks if each detailed requirement is satisfied by the project schedule. For each detailed requirement satisfied by the project schedule a point is earned. Then with equation (2) the value of each Schedule Indicator is found. The weighted sum of each indicator is the Schedule Health $(\mathrm{SH})$ assessment ranking (eq. 1).

If the Schedule Health Assessment is performed prior to project execution for a master schedule, the set of weights related to Indicators one, two and three (Wgm) will be used. In case of evaluation of a detailed schedule the Indicator number four will be included and the related set (Wgd) will apply. If the Schedule Health Assessment is performed in the schedule maintenance phase all Indicators are needed and the related set of weights (Wgc) will apply (fig. 5).

Testing of the proposed method has been carried out in a sample case study covering both the detailed planning and the controlling phases. A simple detailed schedule of a construction project of a shopping centre has been tested. The construction schedule was developed with MS Project ${ }^{\circledR}$. The network is composed of 148 activities and a fragnet of the sample schedule can be found in fig. 6 . A fragnet is a network fragment, or a portion of the project schedule that relates to a specific project phase. First the construction detailed schedule was evaluated, and the encompassed Indicators were "General Requirements", "Construction Process", "Schedule Mechanics" and "Cost and Resources". Thus the Wgd set of weights was used. Then 
the Health Assessment was performed for the control phase and schedule maintenance, thus involving all of five indicators and the use of the $W g c$ set of weights (figure 5).

$<$ Please insert figure 6 here $>$

$<$ Please insert figure 7 here $>$

In the planning phase the applied weights (Wgdi) for the detailed schedule are computed on a set of only 64 requirements. Applying equations (1) and (2) the final grade $\mathrm{SH}$ achieved by the schedule was found. The $\mathrm{SH}$ value was $67 \%$. The table 1 shows the report scoresheet of the Schedule Health Assessment procedure for the sample project and figure 8 summarizes the results with a performance graph.

The result obtained by the schedule was good enough for project management purposes, but suffered from a set of deficiencies. The "General Requirements" indicator earned 13 positive points out of 17 , thus showing a very good schedule and activity definition process though revealing the absence of a standardized schedule process procedure. Also the "Construction Process Requirements" indicator obtained a good score of 10 points out of 11 . In fact almost all the detailed requirements about activity sequencing, activity duration, activity timing and construction process safety and productivity were properly implemented. The schedule health indicator no. 3 , "Schedule Mechanics Requirements" achieved a good grade, 19 points out of 27. Schedule requirements about computerized networking technique implementation were not completely satisfied by sample schedule. Network logic was clear and correctly implemented, but dangling activities were found. Critical path and float definition were properly defined and no activity mis-assignments were found. Instead 
a number of negative lags (leads) and constraints were detected. No monetary value and few resources have been loaded, so only one point was earned by the schedule for the "Cost and Resources Requirements" indicator, because of the satisfaction of the detailed requirement about resource conflicts analysis.

Above all, most of the schedule quality requirements of the planning phase were considered fulfilled by the sample schedule, as shown in the graph of figure 8 .

$<$ Please insert table 1 here $>$

$<$ Please insert table 2 here $>$

In the controlling phase the Schedule Health Assessment procedure was developed with the complete check list of the 75 requirements for schedule maintenance. The "Control Process Requirements" indicator earned 8 points out of 11. Most of the schedule quality requirements were considered fulfilled by the sample schedule, and no invalid dates and missed tasks were found. Applying equations (1) and (2) the final grade $\mathrm{SH}$ achieved by the schedule was found. The SH value was $68 \%$. The table 2 shows the report scoresheet of the Schedule Health Assessment developed method for the sample project, and the graph of figure 9 presents schedule health assessment results. A snapshot of the control barchart view of the sample schedule is shown in figure n. 7.

$<$ Please insert figure 8 here $>$

$<$ Please insert figure 9 here $>$ 


\section{Discussion}

The Schedule Health Assessment procedure has been developed for completing the quality assessment of a construction schedule. A project schedule has a crucial importance as for project management and thus these planning outputs are to be properly developed and maintained. Poor implementation of schedule in the construction sector is very common, especially in medium - small size projects. More than this, shortage and limits of network - based programming techniques for construction projects are very well known (Kenley, Seppanen, 2010). Thus improved understanding over the quality of construction project time management processes and relating solutions is seen to be an important component of project management research. A major finding based on the development of Schedule Health Assessment procedure is the identified need to guide the scheduling process, which is needed i) to show the path for schedule preparation and ii) to produce an effective approach for the project time management i.e. schedule implementation and control. The developed Schedule Health Assessment method has the aim of supporting project schedulers in the project planning phase to develop master and detailed schedules, and in the project execution phase, to support the schedule controlling process.

In the planning phase the project team needs to develop a sound and good quality schedule, and the planning process can be supported by following the Schedule Health requirements, first used as guidelines of the project management schedule subprocesses, and then used for quality control purposes at the end of the scheduling process to detect any weakness in the master or detailed schedule thus allowing the 
project scheduler and/or the entire project team to provide a remedy or to correct the schedule. Similarly, in the controlling phase the Schedule Health Assessment method can provide correct information for schedule maintenance.

It was found that the previous studies over construction related scheduling focus mainly on contractual requirements, i.e. have a legal concern on duties and responsibilities of the parties in question, usually owner and contractor. The proposed method, instead, has the purpose of being a driver of the scheduling process, or in other words it can be understood as a schedule management-oriented solution. Beside this, there are many other differences between the proposed method and existing ones: the different number of requirements, the grouping and weighting system, the automatization versus hand-made check-list compilation. Also, the proposed method entails explicitly safety in the construction process requirements, and can be used in each stage of the development of the project schedule (master, detailed and maintenance).

Concerning the different phases of the construction process and their needs, the method was developed for two main purposes, at the contractor's detailed schedule level for the project execution and at the project manager's level once the contractor's schedule has been uploaded and integrated into a master schedule. The weighting system of schedule indicators changes related to the level of application. It is also possible to modify weights from project to project based on project priority. The definition of such project oriented weights will be object of future research.

The Schedule Health Assessment proposed procedure was also developed with the purpose of being a simple method, easy to be performed by construction Small and Medium-sized Enterprises (SME), which need a good quality level schedule but usually have scarce resources for this task. A non - structured interview with the 
company's project scheduler of the sample project revealed a substantial agreement on the results of the proposed method. In fact the final grade of both Health Assessment procedures was almost 70\%, which means a satisfactory / good level of the schedule. The little time amount available for project scheduling process was the main cause of the fair level of performance obtained. But the quality level was in line with the company's existing project practices and was also meeting contract provisions. Metrics for quality of a project schedule should also indicate a threshold of suitability for practical applications, i.e. if the schedule quality level fits for the purpose. Threshold values could change in function of project goals and characteristics. Limit values should be set case by case, cautiously. Future research should also be oriented to schedule metrics settings, always with the aim of defining construction schedule quality guidelines to apply mostly in the scheduling developing process rather than in a schedule evaluation phase.

\section{Conclusions}

A Schedule Health Assessment method has been proposed with the aim of improving the quality of a construction schedule and scheduling process. The quality level of scheduling process and of schedule itself can be measured through five Key Performance Indicators, termed Health Indicators, which are the following: general requirements, construction process requirements, schedule mechanics requirements, cost and resources requirements and control process requirements. The different classes of requirements can be used to measure schedule health related to the different phases of construction project: master schedule, detailed schedule and schedule 
maintenance. The Schedule Health Assessment method introduces check lists of detailed requirements which can be used as a guide to scheduling process itself. In fact the method has also the aim of being a pro-active method for master and detailed construction scheduling i.e. it can be used as a guide in the schedule development process by project planners or for quality assessment for controlling purposes by project supervisors. It is believed that the proposed method has also the effect of increasing project control in the execution phase, as quality audit of the schedule maintenance process can have the effect of enhancing the monitoring and controlling process.

The proposed five Schedule Health Indicators are based on the seventy five detailed requirements identified from pertinent literature and existing standards. A model and relative weights of the Indicators has been developed, and the weighted sum of the performance level of each indicator has been indicated to be the Health Assessment of the evaluated schedule. It is believed that a main result has been the description of the detailed requirements for each Schedule Health Indicator listed in the appendix (table A1, A2, A3, A4, A5), which is seen as main new knowledge contribution. Future research has been planned to address indicators' weight for the evaluation process.

The proposed method was tested on a sample project, providing schedule performance charts for the detailed schedule and for the maintenance schedule process. The result of the Schedule Health Assessment procedure indicated an average quality level of the sample schedule. This means that further improvement of the evaluated schedule was possible, as to increase its schedule health level, but an interview with the company's project scheduler revealed that the evaluated schedule was believed to have the needed quality level. This reveals much insight of real objectives in 
construction project scheduling, as it is believed that another step forward is still needed by project scheduling to become an effective production plan and not only a schedule.

As Schedule Health Assessment is performed through checklists, it is also believed that the developed method is suitable for the majority of owner's consultants and Small and Medium-sized Enterprises of the construction sector, where resource shortage for project planning and scheduling can lead to the development of low quality schedules.

In opposition to the legal connotation of existing Schedule Health Assessment methods, which mainly aim at performing schedule quality assessment for contract management purposes, the proposed method has been developed also to be used as a guide for the scheduling process. Further research is needed in this direction, as a proof of the proposed concept, by multiple project schedules testing.

The proposed method has a strong connotation in the construction sector, or it is construction oriented, while indeed most of existing standards are not. In fact the second indicator, construction process requirements, aims at developing a process oriented project schedule, conforming the schedule to the execution of project work on-site. Particularly the detailed requirements concerning safety and work-flow are believed to be very important, especially in the evaluated sample case of a network based schedule, with the objective of conforming network logic and activity durations to the need of production in the building site without a formally defined Location Breakdown Structure. This is a well-known gap of network-based schedule, but it is believed that the generalization of good practices like Schedule Health Assessment can bridge between process needs and scheduling method. 


\section{REFERENCES}

Association for Project Management, APM (2012) The Scheduling Maturity Model. APM U.K.

Association for Project Management, APM (2015) APM Competence Framework. $2^{\text {nd }}$ edition v. 1.0. APM U.K.

Birrell G. S. (1980) Construction Planning - Beyond the Critical Path, Journal of the Construction Division American Society Civil Engineers, 106(3), 389-407

Bragadin M.A., Kahkonen K., 2014. Schedule Health Assessment for Construction Projects. Proceedings ICEC IX world congress Re-Engineering Total Cost Management, Milan Italy.

Bragadin M.A., Kahkonen K., 2015. Safety, Space and Structure Quality Requirements in Construction Scheduling. Proceedings $8^{\text {th }}$ Nordic Conference on Construction Economics and Organization, Tampere, Finland.

Callahan M.T., Quackenbush D.G., Rowings J.R. (1992) Construction Project Scheduling McGraw-Hill, Boston U.S..

Carson C., Oakander P., Relyea P., (Edited by) (2014). CPM Scheduling for Constructions Best Practices and Guidelines. PMI Project Management Institute Inc. U.S. 
De La Garza J.M., 1990, Knowledge-Elicitation Study in Construction Scheduling Domain. In Journal of Computing in Civil Engineering 4(2), 135-153.

De La Garza J.M., East E.W., Yau N.J., 1990, A Knowledge Engineering Approach to Analysis and Evaluation of Construction Schedules. In USACERL Tecnical Report P-90/07 (USACERL) February 1990.

Douglas E.E. (2006) Recommended Practice No. 14R-90: Responsibility and required skills for a project planning and scheduling professional. AACE International

Douglas E.E. (2009) Recommended Practice No. 48R-06: Schedule constructability review. AACE International

Douglas E.E., Gransberg D.D. (2009) Recommended Practice No. 30R-03: Implementing project constructability. AACE International

Dvir D., Raz T. Shenhar A.J., 2003. An empirical analysis of the relationship between project planning and project success. In International Journal of Project Management 21, 89-95.

Dzeng R-J., Lee H-Y., (2000). Automated Schedule Advisory System. In Proceedings of the $17^{\text {th }}$ ISARC, Taipei, Taiwan. 
Dzeng R-J., Lee H-Y., 2004. Critiquing contractors' scheduling by integrating rulebased and case-based reasoning. In Automation in Construction 13, 665-678.

Dzeng R-J., Tommelein I.D., 2004. Product Modeling to support case-based construction planning and scheduling. In Automation in Construction 13, 341-360.

Fishman I., Levitt R., 2007. The virtual design team and quantum: comparison of project organization models. www.ibico-cor.com (accessed april 2014).

Galloway P.D., 2005. CPM Scheduling and How the Industry Views Its Use. AACE International Transactions.

Gantt H.L., 1913. Work, wages and profit. The Engineering Magazine Co. New York.

Griffith A. F., 2005, Scheduling practices and project success. In AACE International Transactions. (Association for the Advancement of Cost Engineering International).

Harris R. B. (1978) Precedence and Arrows Networking Techniques for Construction Wiley, New York U.S..

Hussain R., Wearne S. (2005), Problems and needs of Project Management in the process and other industries. Chemical Engineering Research and Design, 83 (A4). 
ISO (2005) ISO 9000:2005 Quality management systems - fundamentals and vocabulary (ISO)

Institution of Professional Engineers New Zeland (IPENZ), 2008. Practice note no. 13: Constructability. (IPENZ, Wellington NZ).

International Project Management Association (IPMA), 2006. ICB - IPMA Competence Baseline, Version 3.0. IPMA The Netherlands.

Isaac S., Navon R., 2014. Can project monitoring and control be fully automated?. Construction Management and Economics, vol. 32, no. 6.

Kenley R., 2014. Editorial - Productivity improvement in the construction process. Construction Management and Economics, vol. 32, no. 6.

Kenley R., Seppanen O., 2010. Location - Based Management for construction: planning, scheduling and control, Routledge, U.K.

Laufer A., Tucker R. L., (1987) Is construction project planning really doing its job? A critical examination of focus, role and process. Construction Management and Economics, vol. 5, no. 3.

Lukka K., Kasanen E., (1995),"The problem of generalizability: anecdotes and evidence in accounting research", Accounting, Auditing \& Accountability Journal, Vol. 8 Iss 5 pp. $71-90$ 
Lukka K., (2000),"The constructive research approach" www.metodix.com accessed September 2015.

Moosavi S. F., 2012. Assessment and Evaluation of Detailed Schedules in Building Construction. MSc Thesis, Concordia University, Canada

Moosavi S. F., Moselhi O., 2012. Schedule Assessment and Evaluation. In Proceedings of the Construction Research Congress 2012 (American Association of Civil Engineering ASCE)

Moosavi S. F., Moselhi O., 2014. Review of Detailed Schedules in Building Construction. In Journal of Legal Affairs and Dispute Resolution in Engineering and Construction (ASCE)

O’Brien J. J., Plotnick F. L., 2006, CPM in Construction Management. Sixth Edition, McGraw-Hill U.S.

Paquin J.P., Couillard J., Ferrand D. J., (2000), Assessing and Controlling the Quality of a Project End Product: The Earned Quality Method. IEEE Transactions on Engineering Management, vol. 47, no. 1.

Program Management System Committee (PMSC), 2012, Planning and Scheduling Excellence Guide (PASEG) v. 2.0. National Defense Industrial Association (NDIA). 
Project Management Institute, 2007, Practice Standard for Scheduling. PMI Project Management Institute, Inc. U.S.

Project Management Institute, 2013, A Guide to the Project Management Body of Knowledge. PMI Project Management Institute, Inc. U.S.

Russell A.D., Tran N., Staub-French S., 2014, Searching for value: construction strategy exploration and linear planning. Construction Management and Economics, vol. 32, no. 6.

Russell A.D., Udaipurwala A., 2000, Assessing the Quality of a Construction Schedule. In Proceedings of the Construction Congress VI: Building together for a Better Tomorrow in an Increasingly complex World (American Association of Civil Engineering ASCE).

Rwelamila P.D., Hall K.A., 1995, Total systems intervention: an integrated approach to time, cost and quality management. Construction Management and Economics, vol. 13 , no. 3 .

Uher, T. E., 2003, Programming and Scheduling Techniques, University of New South Wales Press Ltd Australia.

United States Government Accountability Office (GAO), 2009, GAO Cost estimating and assessment guide, GAO-9-.3SP, (U.S. GAO). 
United States Government Accountability Office (GAO), 2012, GAO Schedule Assessment guide, GAO-12-120G, (U.S. GAO).

U.S. Defense Contract Management Agency (DCMA), 2012, Earned Value Management System (EVMS) Program Analysis Pamphlet (PAP). (U. S. Department of Defense DCMA)

Zwikael, O. Globerson S., 2004, Evaluating the quality of project planning: a model and field results. In International J. of Production Research, vol. 42, issue 8, 15451556. 


\section{Appendix}

Table A1 General Requirements.

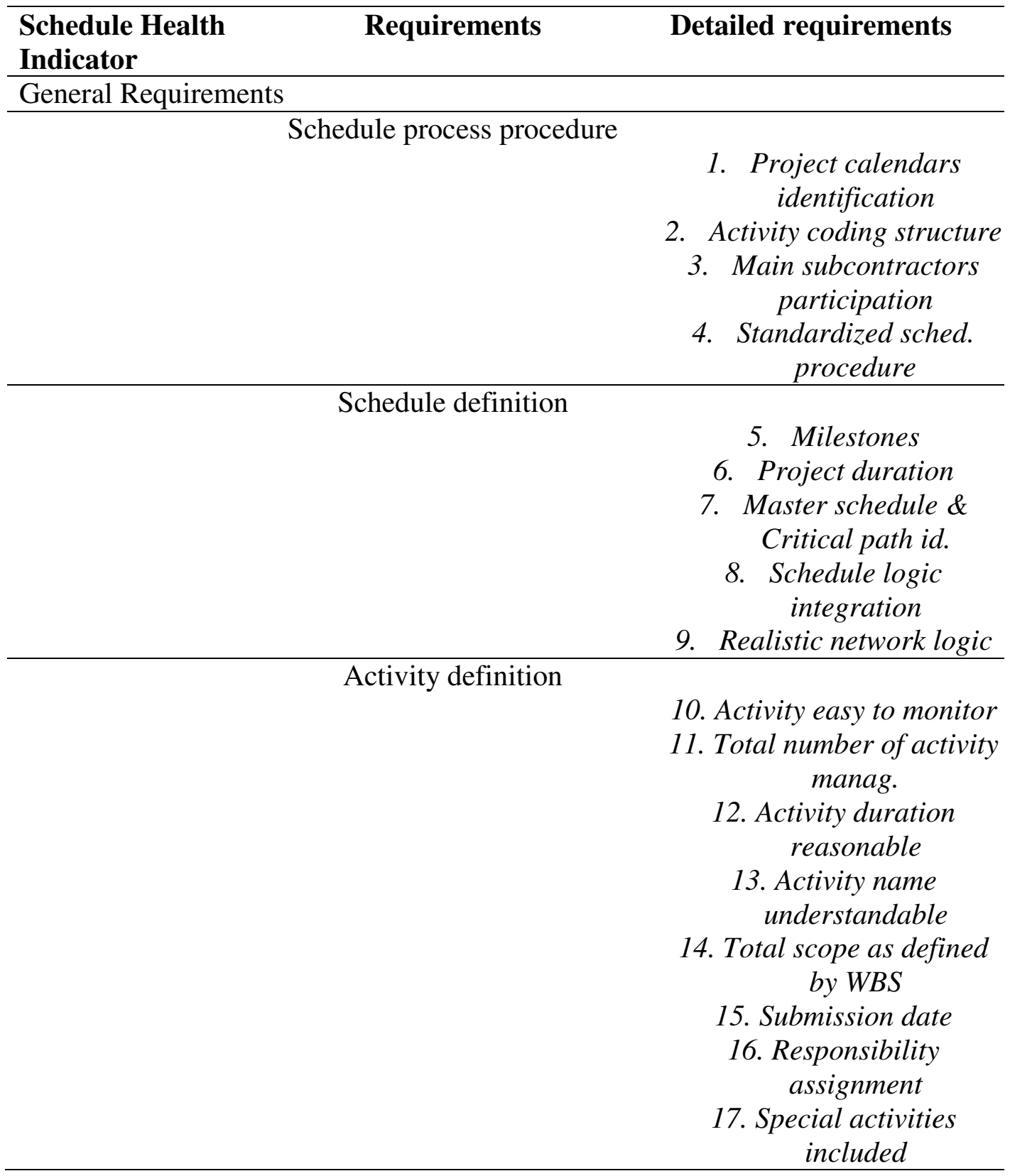


Table A2 Construction Process Requirements.

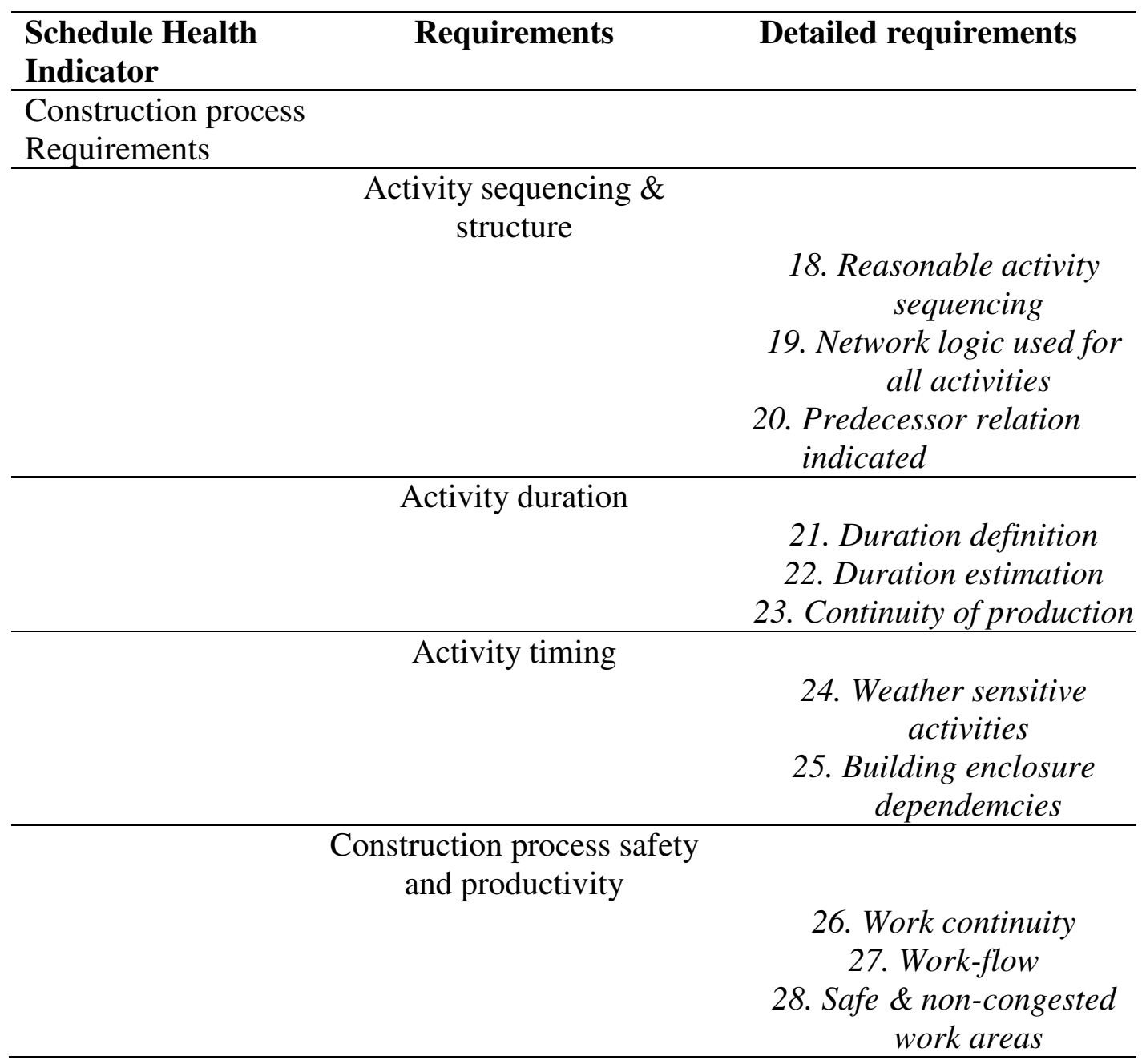


Table A3 Schedule Mechanics Requirements.

\begin{tabular}{|c|c|c|}
\hline $\begin{array}{l}\text { Schedule Health } \\
\text { Indicator }\end{array}$ & Requirements & Detailed requirements \\
\hline $\begin{array}{l}\text { Schedule mechanics } \\
\text { Requirements }\end{array}$ & & \\
\hline & Network and logic & $\begin{array}{l}\text { 29. Open ended activities } \\
\text { 30. Summary tasks logic } \\
\text { 31. Missing logic } \\
\text { 32. Relationship ratio } \\
\text { 33. Relationship types } \\
\text { 34. High duration }\end{array}$ \\
\hline
\end{tabular}

Critical path

35. Critical path \& critical activities

36. Critical activities features

37. Multiple critical paths

38. Critical path test

39. Critical path length index (CPLI)

40. Critical path logic

41. Schedule criticality rate

42. Near criticality rate

43. Critical activity duration

Float

44. Float computation

45. Reasonable float dimensions

46. Excessive total float

47. Negative float

Soft \& Hard Constraints, Buffers

48. ASAP \& ALAP

computation

49. Constraints

50. Number of constraints

51. Buffers

Activity Mis-assignments

52. No activity mis-

assignments

53. No empty milestones

Lag \& Lead (negative lag)

54. Number of lags 55. No leads 
Table A4 Cost and Resources Requirements.

\begin{tabular}{|c|c|c|}
\hline $\begin{array}{l}\text { Schedule Health } \\
\text { Indicator }\end{array}$ & Requirements & Detailed requirements \\
\hline \multicolumn{3}{|l|}{$\begin{array}{l}\text { Cost and Resources } \\
\text { Requirements }\end{array}$} \\
\hline \multicolumn{3}{|c|}{$\begin{array}{c}\text { Monetary value/cost of } \\
\text { activities }\end{array}$} \\
\hline & & $\begin{array}{l}\text { 56. Monetary value of } \\
\text { activities } \\
\text { 57. Total monetary value } \\
\text { 58. Progress payment }\end{array}$ \\
\hline \multicolumn{3}{|c|}{ Project cost ratio } \\
\hline & & 59. Project cost ratio range \\
\hline \multicolumn{3}{|c|}{ Resource loaded activities } \\
\hline & & $\begin{array}{l}\text { 60. Resource loading } \\
\text { 61. Resource productivity } \\
\text { 62. Resource conflicts }\end{array}$ \\
\hline & Project total level of effort & \\
\hline & & $\begin{array}{c}\text { 63. Total amount working } \\
\text { hours/days } \\
\text { 64. Project effort ratio }\end{array}$ \\
\hline
\end{tabular}


Table A5 Control Process Requirements.

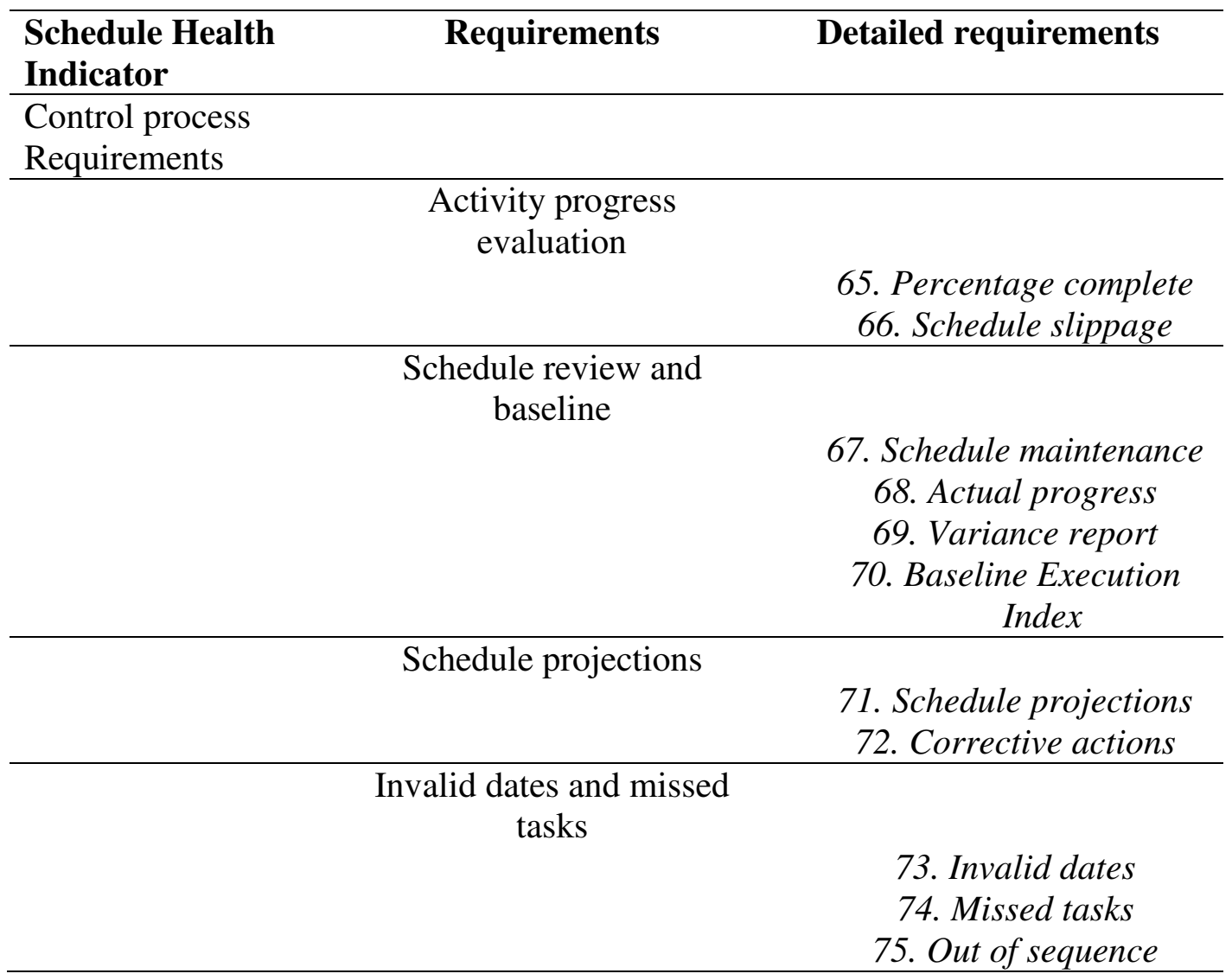


CONSTRUCTION SCHEDULE QUALITY PROBLEM
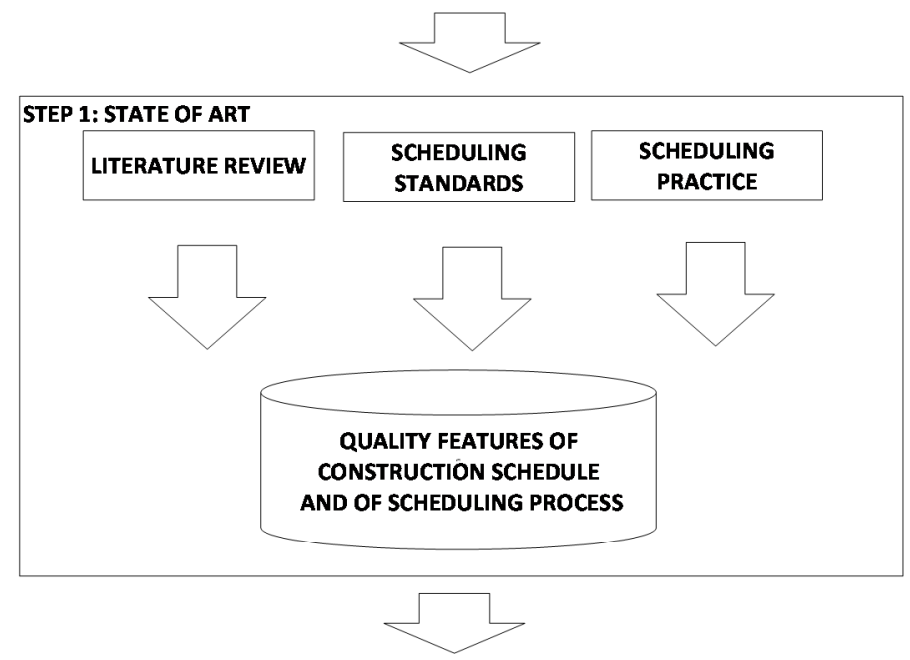

STEP 2: REQUIREMENT ANALYSIS

ANALYSIS AND SELECTION PROCESS OF QUALITY REQUIREMENTS
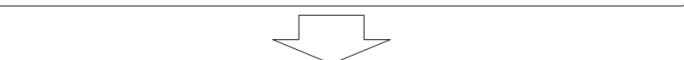
SCHEDULE AND OF SCHEDULING PROCESS

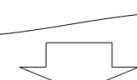

SCHEDULE HEALTH ASSESSMENT

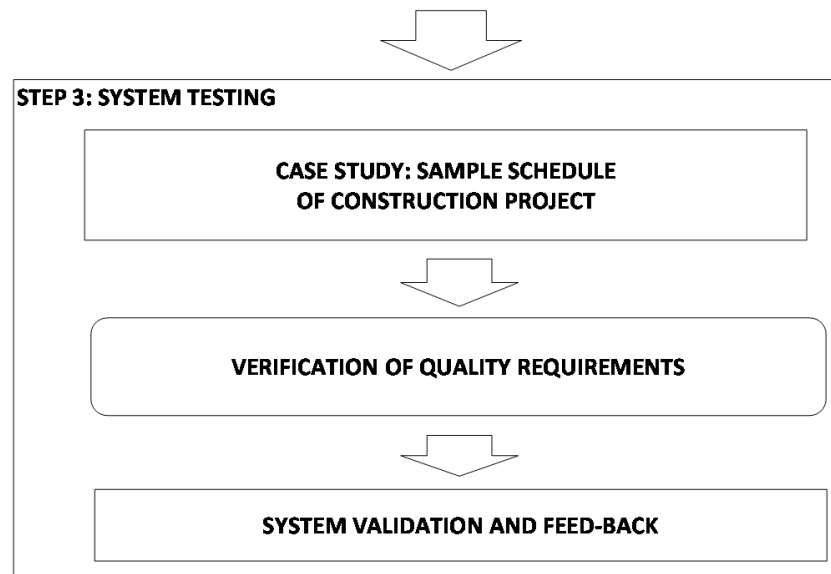

Figure 1 Research procedure 


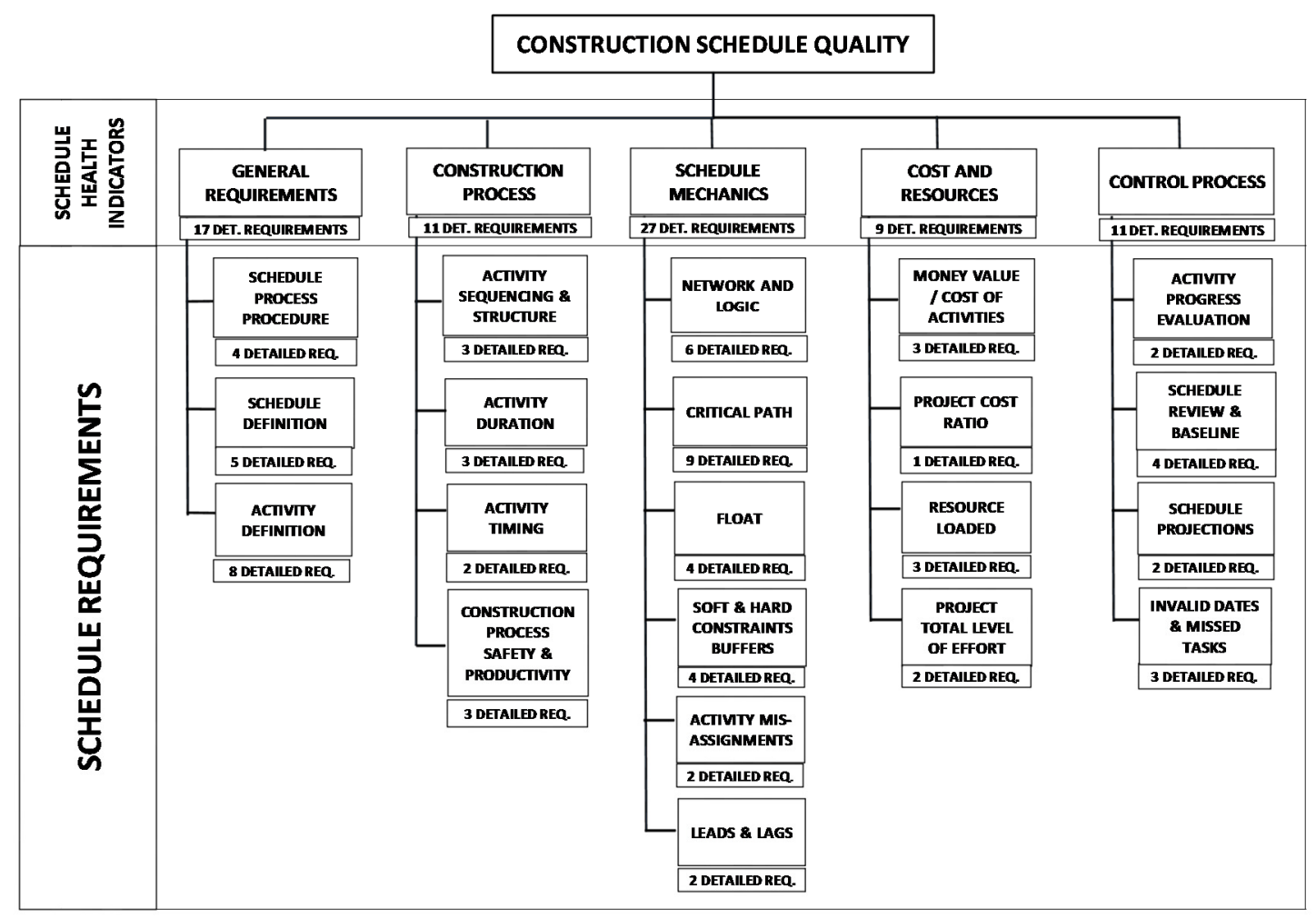

Figure 2 Schedule Health Indicators and Schedule Requirements 
Schedule Health indicator Requirements

RBS

3

3. Schedule mechanics requirements:

a. Network and logic

3.a
Detailed requirements

(specific requirements)

3.a.01 29 Open ended activities (activities without affiliation) should be avoided

Summary tasks with logic relationships should be avoided

Missing logic: there should not be any incomplete tasks with missing logic

Relationships ratio: total number of relationships/total number of activities should

be limited

Relationship types (FS, SS, FF, SF). Start to Finish SF is counter-intuitive, it should be

avoided

High duration: the number of incomplete tasks with high duration should be limited 1 total score score notes

6

Oor 1

Oor 1

oor 1

oor 1

0or 1

Oor 1

Figure 3 Sample checklist of the Schedule Health Assessment proposed method 

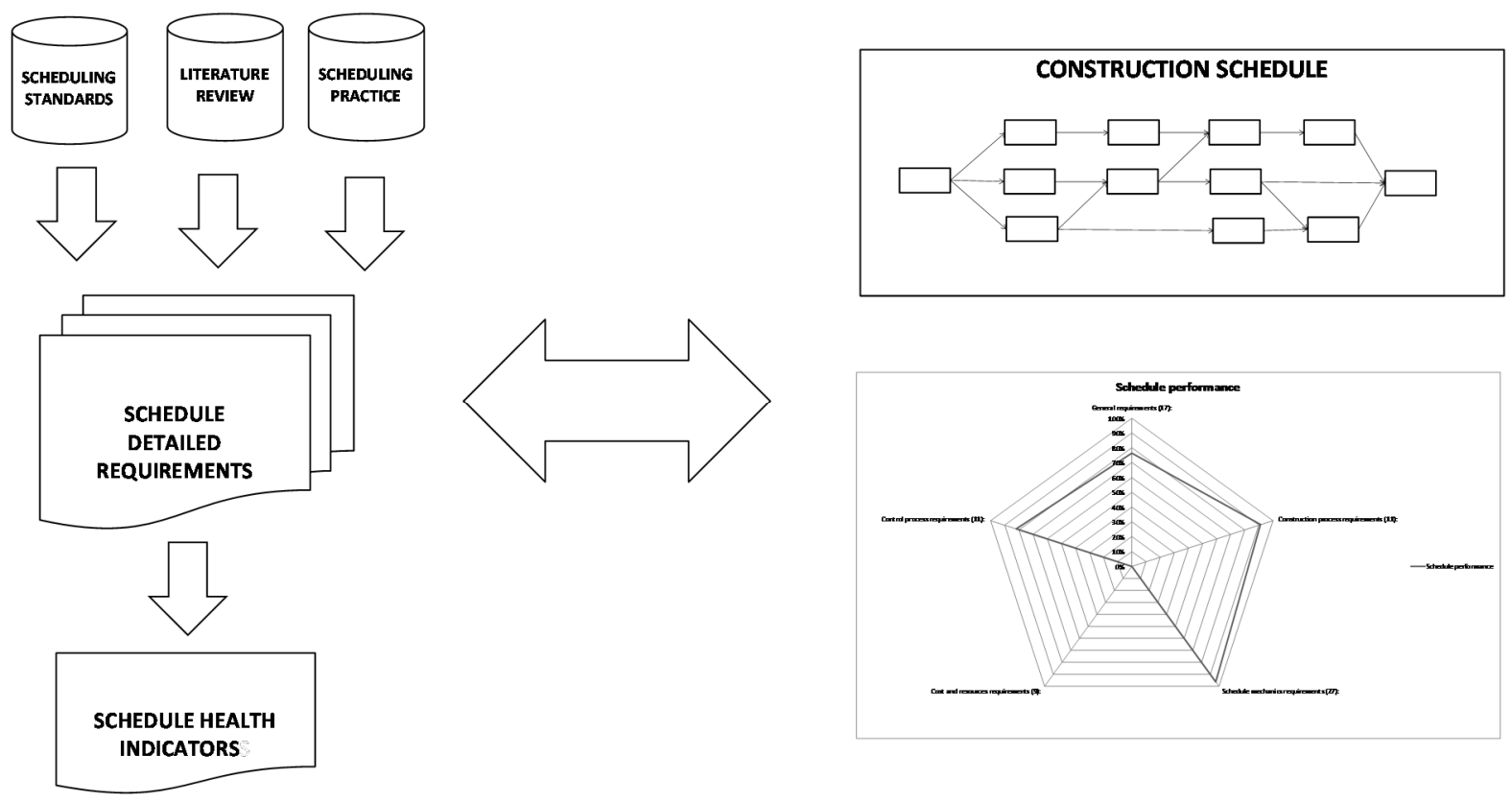

Figure 4 Proposed method framework: Schedule requirements vs Schedule performance 


\begin{tabular}{|c|c|c|c|c|c|c|c|}
\hline & \multicolumn{2}{|c|}{$\begin{array}{l}\text { SCHEDULE HEALTH } \\
\text { INDICATORS: }\end{array}$} & \multirow{2}{*}{$\begin{array}{c}\text { GENERAL } \\
31 \%\end{array}$} & \multirow{2}{*}{$\begin{array}{c}\text { CONSTRUCTION } \\
\text { PROCESS } \\
20 \%\end{array}$} & \multirow{2}{*}{$\begin{array}{c}\text { SCHEDULE } \\
\text { MECHANICS } \\
\\
49 \%\end{array}$} & \multirow{2}{*}{$\begin{array}{c}\text { COST AND } \\
\text { RESOURCE } \\
/\end{array}$} & \multirow{2}{*}{$\begin{array}{c}\text { CONTROL } \\
\text { PROCESS } \\
/\end{array}$} \\
\hline \multirow{3}{*}{ 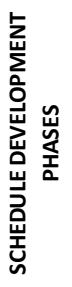 } & \multirow{2}{*}{ 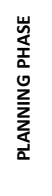 } & $\begin{array}{c}\text { STEP ONE: MASTER } \\
\text { SCHEDULE }\end{array}$ & & & & & \\
\hline & & $\begin{array}{l}\text { STEP TWO: } \\
\text { DETAILED } \\
\text { SCHEDULE }\end{array}$ & $27 \%$ & $17 \%$ & $42 \%$ & $14 \%$ & I \\
\hline & 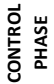 & $\begin{array}{l}\text { STEP THREE: } \\
\text { MAINTENANCE }\end{array}$ & $23 \%$ & $15 \%$ & $36 \%$ & $12 \%$ & $14 \%$ \\
\hline
\end{tabular}

Figure 5 Schedule development phases and related Health Indicators relative weights 


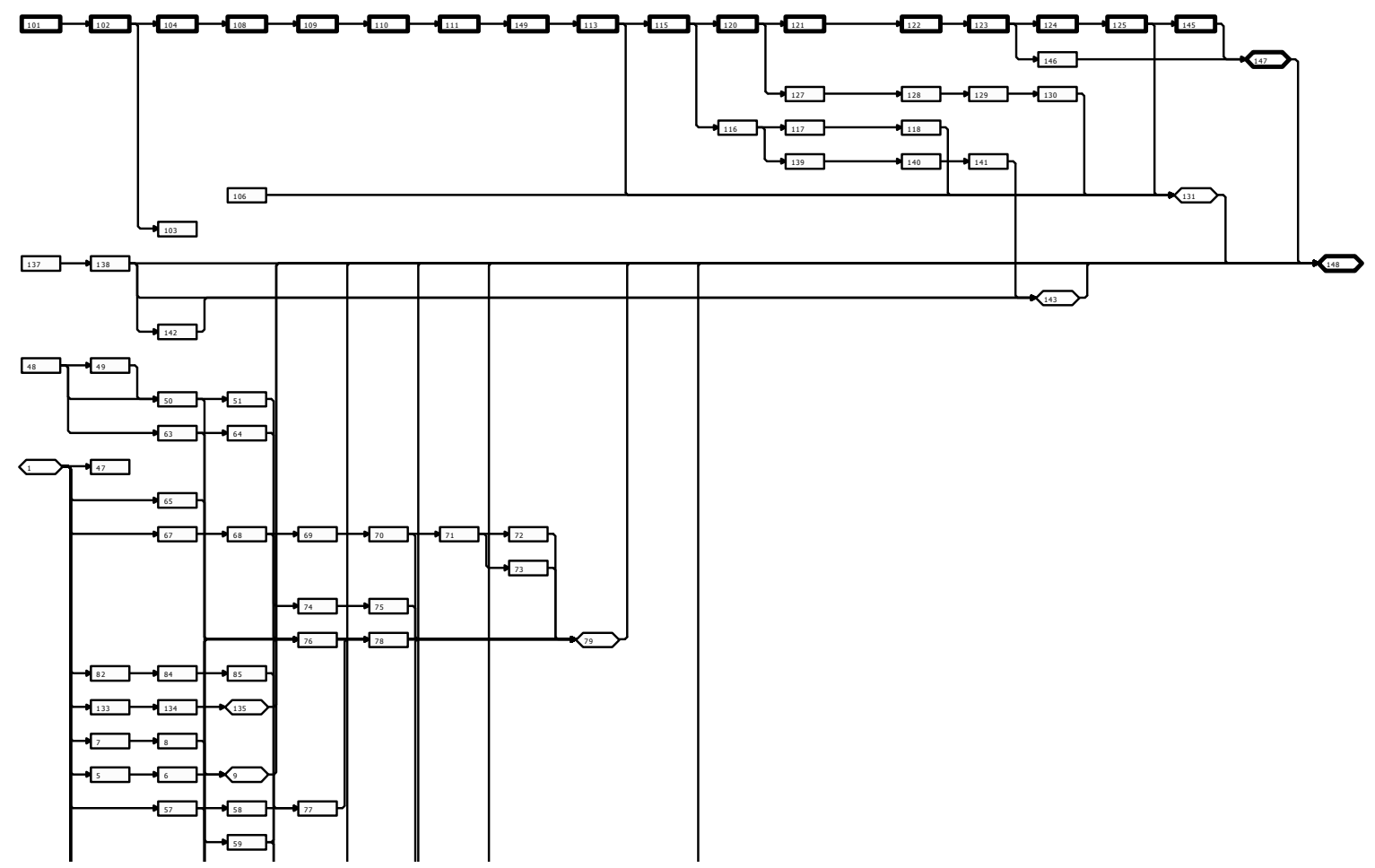

Figure 6 Snapshot of a network fragment of the sample schedule, planning phase detailed schedule (performed with MS Project $®)$ ). 


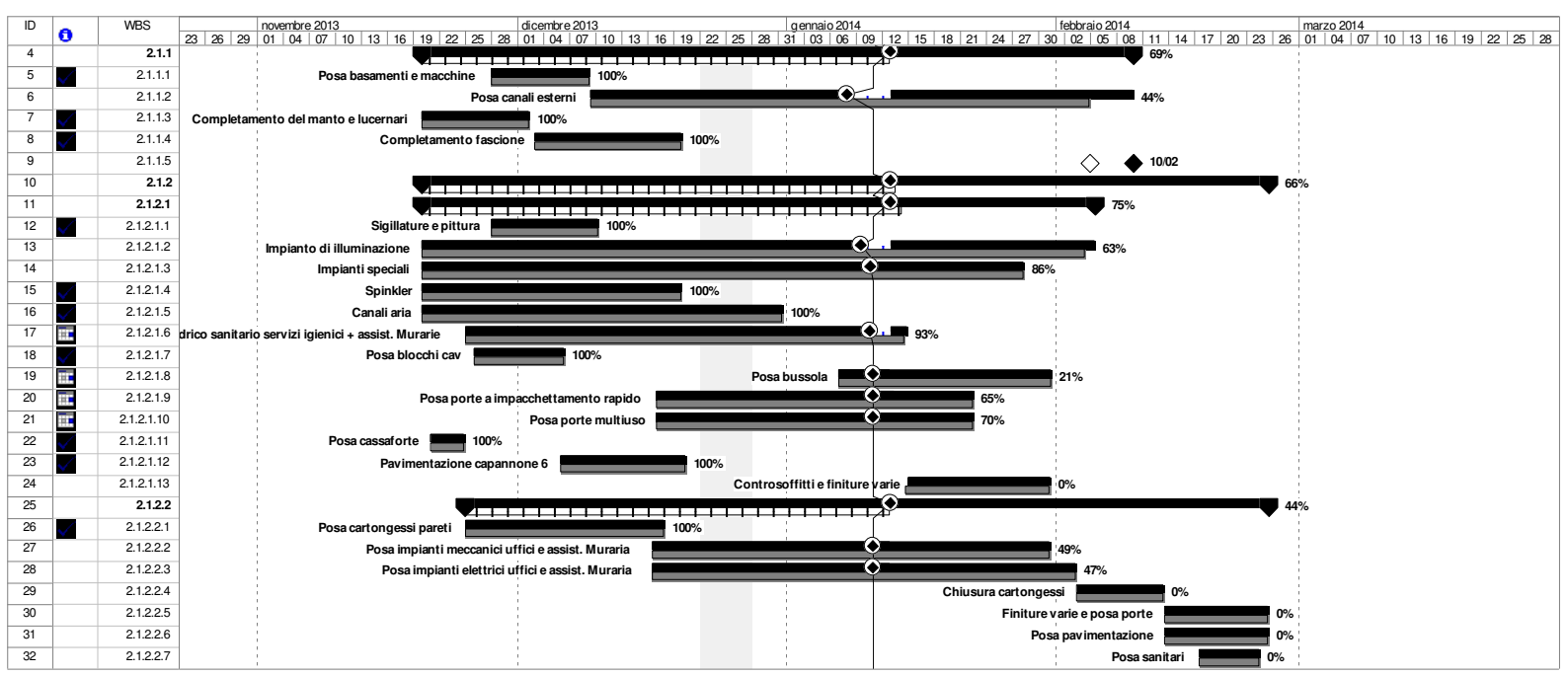

Figure 7 Snapshot from analysed sample schedule, controlling phase (MS Project ${ }^{\circledR}$ ) 


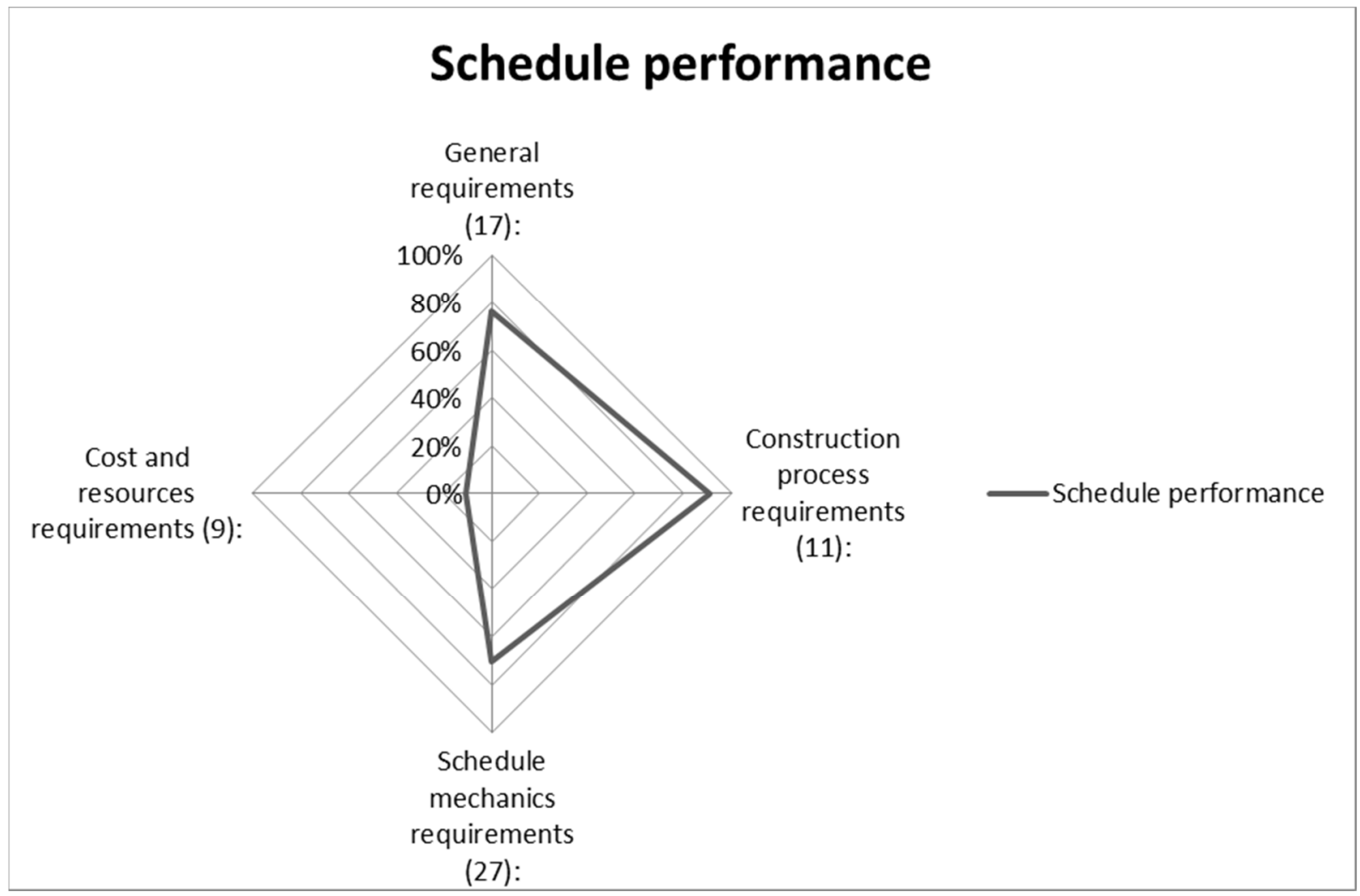

Figure 8 Schedule performance graph of the sample detailed schedule. 


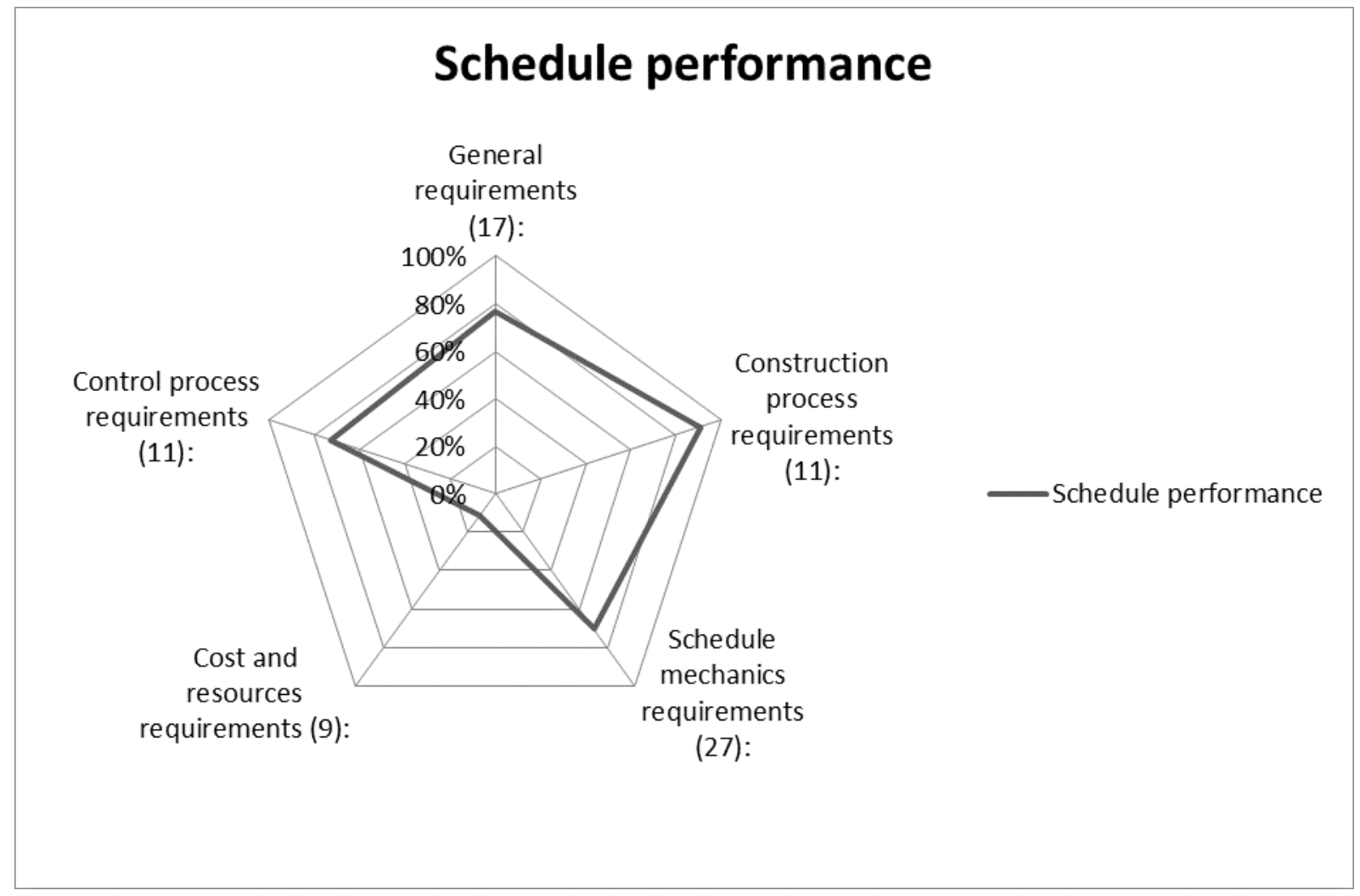

Figure 9 Schedule performance graph of the sample project, controlling phase, maintenance schedule. 
Table 1 Schedule Health Assessment: sample schedule report sheet - planning phase

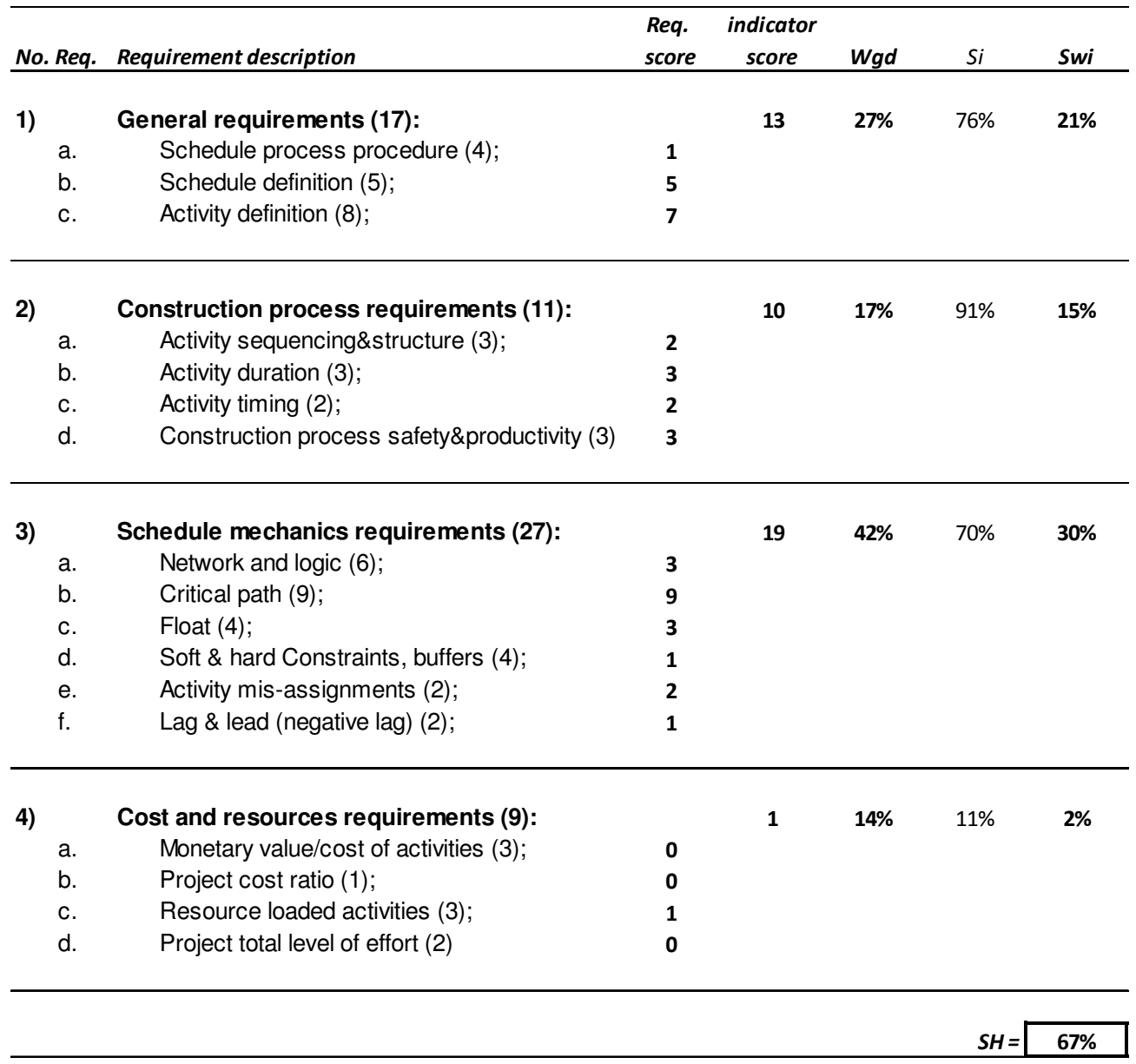


Table 2 Schedule Health Assessment: sample schedule report sheet - controlling phase

\begin{tabular}{|c|c|c|c|c|c|c|}
\hline No. Req. & Requirement description & $\begin{array}{l}\text { Req. } \\
\text { score }\end{array}$ & $\begin{array}{c}\text { indicator } \\
\text { score }\end{array}$ & $W g c$ & Si & Swi \\
\hline $\begin{array}{l}\text { a. } \\
\text { b. } \\
\text { c. }\end{array}$ & $\begin{array}{l}\text { General requirements (17): } \\
\text { Schedule process procedure (4); } \\
\text { Schedule definition (5); } \\
\text { Activity definition (8); }\end{array}$ & $\begin{array}{l}1 \\
5 \\
7\end{array}$ & 13 & $23 \%$ & $76 \%$ & $18 \%$ \\
\hline $\begin{array}{l}\text { a. } \\
\text { b. } \\
\text { c. } \\
\text { d. }\end{array}$ & $\begin{array}{l}\text { Construction process requirements (11): } \\
\text { Activity sequencing \& structure (3); } \\
\text { Activity duration (3); } \\
\text { Activity timing (2); } \\
\text { Construction process safety\&productivity (3) }\end{array}$ & $\begin{array}{l}2 \\
3 \\
2 \\
3\end{array}$ & 10 & $15 \%$ & $91 \%$ & $14 \%$ \\
\hline
\end{tabular}

3) Schedule mechanics requirements (27):

$\begin{array}{lll}\text { a. } & \text { Network and logic (6); } & 3 \\ \text { b. } & \text { Critical path (9); } & 9 \\ \text { c. } & \text { Float (4); } & 3 \\ \text { d. } & \text { Soft \& hard Constraints, buffers (4); } & 1 \\ \text { e. } & \text { Activity mis-assignments (2); } & 2 \\ \text { f. } & \text { Lag \& lead (negative lag) (2); } & 1\end{array}$

4) Cost and resources requirements (9):

$\begin{array}{llllll}\text { a. } & \text { Monetary value/cost of activities (3); } & 0 & 1 & 12 \% & \\ \text { b. } & \text { Project cost ratio (1); } & 0 & 11 \% \\ \text { c. } & \text { Resource loaded activities (3); } & 1 & \\ \text { d. } & \text { Project total level of effort (2) } & \mathbf{0} & \end{array}$

5) Control process requirements (11):

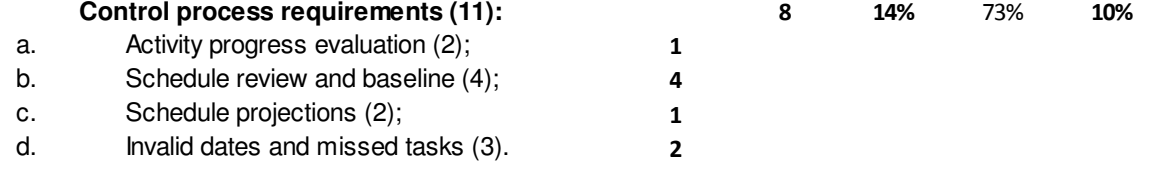




\section{Funding}

This work was supported by Fondazione Cassa di Risparmio in Bologna (CARISBO) - Bologna Italy [progetto 2013/0345]. 